\title{
MEASURES ON PRODUCT SPACES
}

\author{
BY \\ E. O. ELLIOTT
}

Abstract. The theory of regular conditional probability is generalized by replacing a probability measure by a (perhaps non- $\sigma$-finite) outer measure and a resulting measure is obtained on the product space. A Fubini-like theorem is obtained for the integrable functions of this measure and a condition is given for this measure to impart the topological properties of being inner regular and almost Lindelöf to the product space when the component spaces also have these topological properties. Thus some theorems for the Morse-Bledsoe product measure [1] are generalized by methods very similar to those used in their paper on product measures [1].

Introduction. By replacing a regular conditional probability with a regular conditional measure we obtain a means to generate a measure on a product space which is more general than product measure. As a matter of fact, product measure may be viewed as a special case of this construction. It therefore seems natural to ask which theorems in the theory of product measure have analogues in this new setting. Some of the most general results on product measures are contained in the Morse-Bledsoe paper (hereafter referred to as PM). We find that by only slightly modifying their proofs, many of their theorems carry over immediately.

Throughout this paper $R=M \times N$ is a fixed product space, $\mu$ is an outer measure on $M$ and $\mathscr{R}$ is a family of subsets of $N . I_{A}$ is the indicator (or characteristic function) of the set $A$ and $\sigma \mathscr{G}$ is the union $\left(\bigcup_{B \in \mathscr{G}} \beta\right)$ of the family $\mathscr{G}$. We assume $\sigma \mathscr{R} \in \mathscr{R}$.

A regular conditional measure $\nu$ is defined as a function on $M \times \mathscr{R}$ such that

(1) for each $x \in M, v(x, \cdot)$ is an outer measure under which the members of $\mathscr{R}$ are measurable, and

(2) for each $\beta \in \mathscr{R}$ the function $\nu(\cdot, \beta)$ on $M$ is $\mu$ integrable (i.e., $\left.\int \nu(x, \beta) \mu d x \leqq \infty\right)$.

For such a regular conditional measure (rcm) $\nu$ we call a rectangle $\alpha \times \beta$ basic provided

$$
\int I_{\alpha}(x) \nu(x, \beta) \mu d x<\infty
$$

and $\alpha$ is $\mu$ measurable and $\beta \in \mathscr{R}$.

A set $C \subset R$ is called a nilset provided

$$
\int I_{C}(x, y) \nu(x, \cdot) d y \mu d x=0
$$

We let $\mathscr{F}=\{C: C$ is a basic rectangle, or $C$ is a nilset $\}$, and take $\tau$ to be the function on $\mathscr{F}$ such that

$$
\tau(C)=\int I_{C}(x, y) \nu(x, \cdot) d y \mu d x
$$

Received by the editors September 27, 1966. 
Now we can define an outer measure $\phi$ on $R$ using $\tau$ as a gauge and $\mathscr{F}$ as a covering family i.e., for any set $S \subset R$,

$$
\phi(S)=\inf _{\mathscr{G} \subset \mathscr{F}, s \subset \sigma \mathscr{G} ; \mathscr{G} \text { countable }} \sum_{C \in \mathscr{G}} \tau(C) .
$$

We shall make implicit use of the following lemma in the proofs of several theorems.

LEMMA. If $S \in \mathscr{F}$ then as a function of $x, \int I_{S}(x, y) \nu(x, \cdot) d y$ is $\mu$ measurable and equal to $\nu(x, S)$, and $I_{S}(x, y)$ is a $\nu(x, \cdot)$ measurable function for $\mu$ almost all $x$.

In the remainder of the paper, we assume $\mathscr{R}$ is a subtractable (i.e., $\beta-\beta^{\prime} \in \mathscr{R}$ whenever $\beta \in \mathscr{R}$ and $\beta^{\prime} \in \mathscr{R}$ ). As a consequence $\mathscr{R}$ is also intersective (i.e., $\beta \cap \beta^{\prime} \in \mathscr{R}$ whenever $\beta \in \mathscr{R}$ and $\left.\beta^{\prime} \in \mathscr{R}\right)$.

1. A Fubini theorem. The fundamental properties of $\phi$ are given in

1.0. THEOREMS.

1. If $S \in \mathscr{F}$ then $0 \leqq \tau(S)<\infty$.

2. $\phi$ is an outer measure on $R$.

3. If $S \in \mathscr{F}$ then $\phi(S) \leqq \tau(S)$.

4. If $T \subset S$ and $S$ is $a$ nilset then $\tau(T)=0$ and $T$ is $a$ nilset.

5. If $A$ is $\mu$ measurable and $B \in \mathscr{R}$, and $S \in \mathscr{F}$ then $(A \times B) \cap S \in \mathscr{F}$.

1.1. THEOREM. $A \times B$ is $\phi$ measurable whenever $A$ is $\mu$ measurable and $B \in \mathscr{R}$.

Proof. Let $S=A \times B, S^{\prime}=(M-A) \times B, S^{\prime \prime}=M \times(N-B)$, note that $R=S \cup S^{\prime} \cup S^{\prime \prime}$ and that $S, S^{\prime}$, and $S^{\prime \prime}$ are disjoint and complete the proof in two parts.

PART I. If $\gamma \in \mathscr{F}$ then $\tau(\gamma)=\tau(\gamma S)+\tau\left(\gamma S^{\prime}\right)+\tau\left(\gamma S^{\prime \prime}\right)$.

Proof. First suppose $\gamma=a \times b$ where $a$ is $\mu$ measurable, $b \in \mathscr{R}$, and $\gamma$ is basic. Clearly

Since for each $z \in R$,

$$
\gamma S=(a A) \times(b B), \quad \gamma S^{\prime}=(a-A) \times(b B), \quad \gamma S^{\prime \prime}=a \times(b-B) .
$$

we obtain with the aid of 1.0.5 that

$$
I_{\gamma}(z)=I_{(\gamma S)}(z)+I_{\left(\gamma S^{\prime}\right)}(z)+I_{\left(\gamma S^{\prime \prime}\right)}(z)
$$

$$
\begin{aligned}
\tau(\gamma)= & \iint I_{\gamma}(x, y) \nu(x, \cdot) d y \mu d x \\
= & \iint\left\{I_{(\gamma S)}(x, y)+I_{\left(\gamma S^{\prime}\right)}(x, y)+I_{\left(\gamma S^{\prime \prime}\right)}(x, y)\right\} \nu(x, \cdot) d y \mu d x \\
= & \int\left\{\int I_{(\gamma S)}(x, y) \nu(x, \cdot) d y+\int I_{\left(\gamma S^{\prime}\right)}(x, y) \nu(x, \cdot) d y\right. \\
& \left.+\int I_{\left(\gamma S^{\prime \prime}\right)}(x, y) \nu(x, \cdot) d y\right\} \mu d x \\
= & \iint I_{(\gamma S)}(x, y) \nu(x, \cdot) d y \mu d x+\iint I_{\left(\gamma S^{\prime}\right)}(x, y) \nu(x, \cdot) d y \mu d x \\
& +\iint I_{\left(\gamma S^{\prime \prime}\right)}(x, y) \nu(x, \cdot) d y \mu d x \\
= & \tau(\gamma S)+\tau\left(\gamma S^{\prime}\right)+\tau\left(\gamma S^{\prime \prime}\right) .
\end{aligned}
$$


Next, if $\gamma$ is a nilset the conclusion is a consequence of 1.0.4.

PART II. $S$ is $\phi$ measurable.

Proof. Suppose $r>0$ and $\phi(T)<\infty$. Choose such a countable subfamily $\mathscr{G}$ of $\mathscr{F}$ that $T \subset \sigma \mathscr{G}$ and $\sum_{\gamma \in \mathscr{G}} \tau(\gamma) \leqq \phi(T)+r$. Using the fact that $R-S \subset S^{\prime} \cup S^{\prime \prime}, 1.0 .3$, 1.0.5, Part I we infer

$$
\begin{aligned}
\phi(T) & \leqq \phi(T S)+\phi(T \sim S) \\
& \leqq \phi(T S)+\phi\left(T S^{\prime}\right)+\phi\left(T S^{\prime \prime}\right) \\
& \leqq \sum_{\gamma \in \mathscr{G}} \phi(\gamma S)+\sum_{\gamma \in \mathscr{G}} \phi\left(\gamma S^{\prime}\right)+\sum_{\gamma \in \mathscr{G}} \phi\left(\gamma S^{\prime \prime}\right) \\
& \leqq \sum_{\gamma \in \mathscr{G}} \tau(\gamma S)+\sum_{\gamma \in \mathscr{G}} \tau\left(\gamma S^{\prime}\right)+\sum_{\gamma \in \mathscr{G}} \tau\left(\gamma S^{\prime \prime}\right) \\
& \leqq \sum_{\gamma \in \mathscr{G}}\left\{\tau(\gamma S)+\tau\left(\gamma S^{\prime}\right)+\tau\left(\gamma S^{\prime \prime}\right)\right\} \\
& \leqq \sum_{\gamma \in \mathscr{G}} \tau(\gamma) \leqq \phi(T)+r .
\end{aligned}
$$

The arbitrary nature of $r$ assures us that

$$
\phi(T)=\phi(T S)+\phi(T-S) .
$$

1.2. THEOREM. If $S \in \mathscr{F}$ then $S$ is $\phi$ measurable.

Proof. If $S$ is a nilset use 1.0.4 and 1.0.3 to see that $\phi(S)=0$; otherwise use 1.1.

1.3. TheOREM. If $S \in \mathscr{F}$ then $\phi(S)=\tau(S)<\infty$.

Proof. From 1.0.1 we know $\tau(S)<\infty$. Hence, by 1.0.3 $\phi(S)<\infty$, and for $r>0$ we may choose such a countable family $\mathscr{G}$ of $\mathscr{F}$ that $S \subset \sigma \mathscr{G}$ and $\sum_{\gamma \in \mathscr{G}} \tau(\gamma) \leqq \phi(S)+r$. Since for each $z \in R, 0 \leqq I_{S}(z) \leqq \sum_{y \in \mathscr{G}} I_{y}(z)$ we may infer,

$$
\begin{aligned}
r+\phi(S) & \geqq \sum_{\gamma \in \mathscr{G}} \tau(\gamma)=\sum_{\gamma \in \mathscr{G}} \iint I_{\gamma}(x, y) \nu(x, \cdot) d y \mu d x \\
& =\int \sum_{\gamma \in \mathscr{G}} \int I_{\gamma}(x, y) \nu(x, \cdot) d y \mu d x \\
& =\iint \sum_{\gamma \in \mathscr{G}} I_{\gamma}(x, y) \nu(x, \cdot) d y \mu d x \\
& \geqq \iint I_{S}(x, y) \nu(x, \cdot) d y \mu d x=\tau(S) .
\end{aligned}
$$

Let us say that $f$ is a simple function if and only if

(1) $f$ takes on at most a countable number of values $v_{i}\left(0 \leqq v_{i} \leqq \infty\right)$, and

(2) the constant sets $f^{-1}\left(v_{i}\right)$ belong to $\mathscr{F}$.

For simple functions we have the following

1.4. THEOREM. If $f$ is a simple function then

$$
\int f(z) \phi d z=\iint f(x, y) \nu(x, \cdot) d y \mu d x
$$


Proof. Write $f(z)=\sum_{\gamma \in \mathscr{S}}\left\{v_{\gamma} I_{\gamma}(z)\right\}$, and use 1.3 and integration by summation.

1.5. THEOREM. If $f(z)=g(z)$ a.e. $(\phi)$, then

$$
\iint f(x, y) \nu(x, \cdot) d y \mu d x=\iint g(x, y) \nu(x, \cdot) d y \mu d x
$$

Proof. Let $T=\{z: z \in R$ and $f(z) \neq g(z)\}$ and in accordance with PM 4.6 choose a sequence $h$ of simple functions so that for each $n I_{T}(z) \leqq h_{n}(z)$ for each $z \in R$ and .1

$$
\int h_{n}(z) \phi d z \leqq 2^{-n}
$$

Let $h^{\prime}$ be the function on $R$ defined by

$$
h^{\prime}(z)=\lim _{n} \inf h_{n}(z)
$$

Using .1, 1.4 and Fatou's lemma we have

$$
\begin{aligned}
0 & =\lim _{n} \inf \int h_{n}(z) \phi d z \\
& =\liminf _{n} \iint h_{n}(x, y) \nu(x, \cdot) d y \mu d x \\
& \geqq \int \lim _{n} \inf \int h_{n}(x, y) \nu(x, \cdot) d y \mu d x \\
& \geqq \iint h^{\prime}(x, y) \nu(x, \cdot) d y \mu d x \geqq 0 .
\end{aligned}
$$

Moreover, if $x \in M$ and $y \in N$ then $I_{T}(x, y) \leqq h^{\prime}(x, y)$. Thus,

$$
\begin{gathered}
\left\{\int h^{\prime}(x, y) \nu(x, \cdot) d y=0\right\} \text { a.e. }(\mu), \\
\left\{h^{\prime}(x, y)=0 \text { a.e. }(\nu(x, \cdot))\right\} \text { a.e. }(\mu), \\
\left\{I_{T}(x, y)=0 \text { a.e. }(\nu(x, \cdot))\right\} \text { a.e. }(\mu), \\
\{f(x, y)=g(x, y) \text { a.e. }(\nu(x, \cdot))\} \text { a.e. }(\mu), \\
\left\{\int f(x, y) \nu(x, \cdot) d y=\int g(x, y) \nu(x, \cdot) d y\right\} \text { a.e. }(\mu), \\
\iint f(x, y) \nu(x, \cdot) d y \mu d x=\iint g(x, y) \nu(x, \cdot) d y \mu d x .
\end{gathered}
$$

We come now to our Fubini-like

1.6. THEOREM. If $f$ is $\phi$ integrable $\left({ }^{1}\right)$ then

$$
\int f(z) \phi d z=\iint f(x, y) \nu(x, \cdot) d y \mu d x
$$

(1) $-\infty \leqq \int f(z) \phi d z \leqq \infty$ and $\{z: f(z) \neq 0\}$ is $\sigma$-finite under $\phi$. 
Proof. Use PM 4.10 to secure such simple functions $g$ and $h$ that $f(z)=g(z)-h(z)$ a.e. $(\phi)$ and

$$
\int f(z) \phi d z=\int g(z) \phi d z-\int h(z) \phi d z .
$$

Thus with the help of 1.4 and 1.5 we infer

$$
\begin{aligned}
\int f(z) \phi d z & =\int g(z) \phi d z-\int h(z) \phi d z \\
& =\iint g(x, y) \nu(x, \cdot) d y \mu d x-\iint h(x, y) \nu(x, \cdot) d y \mu d x \\
& =\int\left[\int g(x, y) \nu(x, \cdot) d y-\int h(x, y) \nu(x, \cdot) d y\right] \mu d x \\
& =\iint[g(x, y)-h(x, y)] \nu(x, \cdot) d y \mu d x \\
& =\iint f(x, y) \nu(x, \cdot) d y \mu d x .
\end{aligned}
$$

2. Topological measures. We now assume that $\mathscr{M}$ is a topology (family of open sets) on $M$ and $\mathscr{N}$ is a topology on $N$ and that $\mathscr{T}$ is the product topology (of $\mathscr{M}$ and $\mathscr{N}$ ) on $R$. We further assume that the members of $\mathscr{M}$ are $\mu$ measurable and that the members of $\mathscr{N}$ are $\nu(x, \cdot)$ measurable for each $x \in M$. Our purpose is to demonstrate that when certain topological properties are possessed by $\mu$ and $\nu$ they are passed on to $\phi$.

We begin by defining these properties in a general setting. Suppose $S$ is a topological space with $\mathscr{S}$ being its family of open sets, and let $\psi$ be a measure on $S$ for which the open sets are measurable.

$\mathscr{S}$ is said to be almost Lindelöf (a.L.) provided that from each covering of $S$ by open sets a countable subfamily can be extracted which covers almost all of $S$.

$\mathscr{S}$ is inner regular (i.r.) provided each open set can be approximated in measure by closed subsets of finite measure, i.e., for each $T \in \mathscr{S}$,

$$
\psi(T)=\sup _{C \subset T, \psi(C)<\infty ; C \text { closed }} \psi(C) .
$$

When these properties are applied to $\mathscr{N}$ it is implied that they hold under each of the measures $\nu(x, \cdot),(x \in M)$.

2.0. Lemma. If $\mathscr{M}$ is a.L. and i.r. and $\mathscr{N}$ is a.L. and i.r., each member of $\mathscr{G}$ is an open rectangle in $R, S=\sigma \mathscr{G}$ and

then

$$
\sup _{\mathscr{H} \subset \mathscr{G} ; \mathscr{H} \text { countable }} \phi(\sigma \mathscr{H})=b<\infty,
$$

$$
\iint I_{S}(x, y) \nu(x, \cdot) d y \mu d x=b .
$$

Proof. So choose a countable subfamily $\mathscr{H}$ of $\mathscr{G}$ that $\phi(\sigma \mathscr{H})=b$, let $T=\sigma \mathscr{H}$ and divide the remainder of the proof into 5 parts. (Let us adopt the notation that if $K \subset R$ and $x \in M$ then $K_{x}=\{y:(x, y) \in K\}$.) 
PART I. $b=\int \nu\left(x, T_{x}\right) \mu d x$.

Proof. Use 1.6.

In Parts II and III we make use of a notion of relative nullity given in PM 6.9. For our purpose it may be condensed as follows: $B$ is null w.r.t. $A$ if and only if for each $x \in B$ there is an open set $\alpha(\in \mathscr{M})$ containing $x$ such that $\mu(\alpha A)=0$.

PART II. If $0<r<\infty$,

$$
\begin{aligned}
A & =\left\{x: \nu\left(x, T_{x}\right)<r<\nu\left(x, S_{x}\right)\right\}, \\
A^{\prime} & =\left\{x: r<\nu\left(x, S_{x}\right)\right\},
\end{aligned}
$$

then $A^{\prime} \in \mathscr{M}$ and $A^{\prime}$ is null w.r.t. $A$.

Proof. Let $t \in A^{\prime}$. Since $r<\nu\left(t, S_{t}\right), S_{t} \in \mathscr{N}, \mathscr{N}$ is i.r., we can and do choose such a closed set $C^{\prime \prime}$ that $C^{\prime \prime} \subset S_{t}, r<\nu\left(t, C^{\prime \prime}\right)<\infty$.

Let $\mathscr{G}_{1}=\left\{\gamma: \gamma \in \mathscr{G}\right.$ and $\left.\gamma_{t} \neq 0\right\}$, check that $C^{\prime \prime} \subset \bigcup_{\gamma \in \mathscr{S}_{1}} \gamma_{t}$ and use the fact that $\mathscr{N}$ is a.L. to secure such a countable subfamily $\mathscr{G}_{1}^{\prime}$ of $\mathscr{G}_{1}$ that $\nu\left(t, C^{\prime \prime}-\bigcup_{\gamma \in \mathscr{G}_{1}^{\prime}} \gamma_{t}\right)=0$.

We may infer from the above that $\nu\left(t, \bigcup_{y \in \mathscr{G}_{1}^{\prime}} \gamma_{t}\right) \geqq \nu\left(t, C^{\prime \prime}\right)>r$ and then secure such a finite subfamily $\mathscr{G}_{1}^{\prime \prime}$ of $\mathscr{G}_{1}^{\prime}$ that

$$
r<\nu\left(t, \bigcup_{\gamma \in \mathscr{G}_{1}^{*}} \gamma_{t}\right)
$$

For each $\gamma \in \mathscr{G}_{1}^{\prime \prime}$ let $\alpha^{\gamma}$ be the member of $\mathscr{M}$ for which $\gamma=\alpha^{\gamma} \times \gamma_{t}$ and take

$$
\begin{aligned}
& \xi=\bigcap_{\gamma \in \mathscr{G}_{1}^{\prime \prime}} \alpha^{\gamma}, \\
& \eta=\bigcup_{\gamma \in \mathscr{G}_{1}^{\prime \prime}} \gamma_{t} ; \\
& \lambda=\xi \times \eta .
\end{aligned}
$$

Note that $t \in \xi \in \mathscr{M}$ and complete the proof in Step 4 below by verifying that $\mu(\xi A)=0$ and $\xi \subset A^{\prime}$.

Step 1. $\lambda \subset \sigma \mathscr{G}_{1}^{\prime \prime}$.

Proof.

$$
\begin{aligned}
\lambda & =\bigcup_{\gamma \in \mathscr{G}_{1}^{\prime \prime}}\left(\xi \times \gamma_{t}\right) \subset \bigcup_{\gamma \in \mathscr{G}_{1}^{\prime \prime}}\left(\alpha^{\gamma} \times \gamma_{t}\right) \\
& =\bigcup_{\gamma \in \mathscr{G}_{1}^{\prime \prime}} \gamma=\sigma \mathscr{G}_{1}^{\prime \prime} .
\end{aligned}
$$

Step 2. $\nu(t, \eta)>r$.

Proof. Use (2) and (1).

Step 3. $\phi(\lambda-T)=0$.

Proof. Let $\mathscr{H}^{\prime}=\mathscr{H} \cup \mathscr{G}_{1}^{\prime \prime}$. Thus $\mathscr{H}^{\prime}$ is a countable subfamily of $\mathscr{G}$, and, in the light of Step 1,

$$
\begin{aligned}
b & =\sup _{\mathscr{K} \subset \mathscr{G} ; \mathscr{K} \text { countable }} \phi(\sigma \mathscr{K}) \geqq \phi\left(\sigma \mathscr{H}^{\prime}\right) \\
& =\phi(T)+\phi\left(\sigma \mathscr{H}^{\prime}-T\right) \geqq \phi(T)+\phi\left(\sigma^{\mathscr{G}_{1}^{\prime \prime}}-T\right) \\
& \geqq \phi(T)+\phi(\lambda-T) \\
& =b+\phi(\lambda-T), \quad 0 \leqq \phi(\lambda-T) \leqq b-b=0 .
\end{aligned}
$$


Step 4. $\mu(\xi A)=0$ and $\xi \subset A^{\prime}$.

Proof. For each $t \in \xi$ we infer from Steps 1 and 2 that

$$
r<v(t, \eta) \leqq v\left(t, S_{t}\right)
$$

and hence that $t \in A^{\prime}$. Accordingly $\xi \subset A^{\prime}$.

Since by Step 3

$$
\int \nu\left(x,(\lambda-T)_{x}\right) \mu d x=\phi(\lambda-T)=0,
$$

it follows that

$$
\mu\left(\left\{x: x \in \xi \text { and } \nu\left(x,(\gamma-T)_{x}\right)>0\right\}\right)=0 .
$$

But, because of Step 2,

$$
\begin{aligned}
\xi A & \subset\left\{x: x \in \xi \text { and } \nu\left(x, T_{x}\right)<r\right\} \\
& \subset\left\{x: x \in \xi \text { and } \nu\left(x, T_{x}\right)<\nu(x, \eta)\right\} \\
& =\left\{x: x \in \xi \text { and } \nu\left(x,(\lambda-T)_{x}\right)>0\right\}
\end{aligned}
$$

Consequently $\mu(\xi A)=0$.

PART III. If $0<r<\infty, A=\left\{x: \nu\left(x, T_{x}\right)<r<\nu\left(x, S_{x}\right)\right\}$ then $\mu(A)=0$.

Proof. Let $A^{\prime}=\left\{x: r<\nu\left(x, S_{x}\right)\right\}$. Then we know from Part II that $A \subset A^{\prime} \in \mathscr{M}$ and $A^{\prime}$ is null w.r.t. $A$.

Now if $C$ is a closed subset of $A^{\prime}$ then: because of the almost Lindelöfness of $\mathscr{M}$ (see PM 6.10 and PM 6.11), $\mu(A C)=0$; however $A C=\left\{x: \nu\left(x, T_{x}\right)<r I_{C}(x)\right\}$. Hence $\nu\left(x, T_{x}\right) \geqq r I_{C}(x)$ a.e. $(\mu)$ and because of this and Part $\mathrm{I}$,

$$
\begin{aligned}
\infty & >b=\int \nu\left(x, T_{x}\right) \mu d x \geqq r \cdot \int I_{C}(x) \mu d x=r \cdot \mu(C), \\
\mu(C) & \leqq b / r<\infty .
\end{aligned}
$$

Consequently, since $\mathscr{M}$ is i.r.,

$$
\mu\left(A^{\prime}\right) \leqq b / r<\infty
$$

and using the inner regularity and almost Lindelöfness of $\mathscr{M}$ (see PM 6.12), $\mu(A)=\mu\left(A A^{\prime}\right)=0$.

PART IV. $\nu\left(x, T_{x}\right)=\nu\left(x, S_{x}\right)$ a.e. $(\mu)$

Proof. Let $\mathrm{Ra}$ be the set of positive rational numbers. Clearly

$$
M \cap\left\{x: \nu\left(x, T_{x}\right) \neq \nu\left(x, S_{x}\right)\right\}=\bigcup_{r \in \mathbb{R}}\left\{x: \nu\left(x, T_{x}\right)<r<\nu\left(x, S_{x}\right)\right\}
$$

and the desired conclusion now follows from Part III.

PART V. $\iint I_{S}(x, y) \nu(x, \cdot) d y \mu d x=b$.

Proof. From Part IV, Part I, and the fact that for each $x, S_{x} \in \mathscr{N}$ and $S_{x}$ is $\nu(x, \cdot)$ measurable we infer

$$
\begin{aligned}
\infty>b & =\int \nu\left(x, S_{x}\right) \mu d x \\
& =\iint I_{S}(x, y) \nu(x, \cdot) d y \mu d x .
\end{aligned}
$$


Let us agree to call $\nu$ continuous if the function $\nu(\cdot, \beta)$ is finite and $\mathscr{M}$ continuous for each set $\beta$ which is measurable under all measures $\nu(x, \cdot)$ where $x \in M$. As a sufficient condition for the desired results, the continuity of $\nu$ is employed in the next

2.1. Lemma. If $\mathscr{M}$ is a.L. and i.r., $\mathscr{N}$ is a.L. and i.r., $\nu$ is continuous, $A \in \mathscr{M}$, $B \in \mathscr{N}, S=A \times B, \mu(A)<\infty, \phi(s)<\infty$, and $r>0$, then for some closed subset $C$ of $S$

$$
\phi(S-C)<r \text {. }
$$

Proof. We assume $\mu(A) \neq 0$ since the result is trivial when $\mu(A)=0$. Now for each $x \in A$ let $b_{x}$ be such a closed subset of $B$ that $\nu\left(x, b_{x}\right) \geqq \nu(x, B)-r /(6 \mu(A))$ and let $a_{x}$ be such a member of $\mathscr{M}$ that $x \in a_{x} \subset A$ and $\left|\nu\left(x, b_{x}\right)-\nu\left(t, b_{x}\right)\right| \leqq r /(6 \mu(A))$ whenever $t \in a_{x}$. Hence

$$
\nu\left(t, b_{x}\right) \geqq \nu(x, B)-r /(3 \mu(A))
$$

whenever $x \in A$ and $t \in a_{x}$.

Noting that $\int_{A} \nu(x, B) \mu d x<\infty$, let $\delta, 0<\delta<\infty$, be such a number that

$$
\int I_{\alpha}(x) \nu(x, B) \mu d x<r / 3
$$

whenever $\alpha(\alpha \subset A)$ is a $\mu$ measurable set and $\mu(\alpha)<\delta$. Now secure such a closed subset $A^{\prime}$ of $A$ that

$$
\mu\left(A-A^{\prime}\right)<\delta .
$$

Let $\mathscr{A}=\left\{\alpha: \alpha=a_{x}\right.$ for some $\left.x \in A\right\}$ and secure such a countable subfamily $\mathscr{A}^{\prime}$ of $\mathscr{A}$ that

$$
\mu\left(A^{\prime}-\sigma \mathscr{A}^{\prime}\right)=0
$$

and such a finite subfamily $\mathscr{A}^{\prime \prime}$ of $\mathscr{A}^{\prime}$ that

$$
\mu\left(\sigma \mathscr{A}^{\prime}-\sigma \mathscr{A}^{\prime \prime}\right)<\delta .
$$

Let $\alpha, \beta$, and $x$ be such sequences that

$$
\alpha_{i}=\alpha_{x_{i}} \in \mathscr{A}^{\prime}, \quad \mathscr{A}^{\prime}=\left\{\alpha_{i}\right\},
$$

and $\beta_{i}=b_{x_{i}}$. Now let $B^{\prime}=\sigma\left\{\beta^{\prime}: \beta^{\prime}=\beta_{i}\right.$ for some $i$ s.t. $\left.\alpha_{i} \in \mathscr{A}^{\prime \prime}\right\}$,

$$
\begin{aligned}
C & =A^{\prime} \times B^{\prime}, \quad S_{1}=\left(A-A^{\prime}\right) \times B, \\
S_{2} & =\left(A^{\prime}-\sigma \mathscr{A}^{\prime}\right) \times B, \quad S_{3}=\left(A^{\prime} \cap\left(\sigma \mathscr{A}^{\prime}-\sigma \mathscr{A}^{\prime \prime}\right)\right) \times B,
\end{aligned}
$$

and

$$
S_{4}=\left(A^{\prime} \cap \sigma \mathscr{A}^{\prime \prime}\right) \times\left(B-B^{\prime}\right),
$$

and infer that $S \subset S_{1} \cup S_{2} \cup S_{3} \cup S_{4} \cup C$. Hence

$$
\phi(S) \leqq \phi\left(S_{1}\right)+\phi\left(S_{2}\right)+\phi\left(S_{3}\right)+\phi\left(S_{4} \cup C\right) .
$$


From (3), (5), and (2) we infer $\phi\left(S_{1}\right)<r / 3$ and $\phi\left(S_{3}\right)<r / 3$ and from (4) that $\phi\left(S_{2}\right)=0$. For each $x \in \sigma \mathscr{A}^{\prime \prime}$ let

$$
d(x)=\sigma\left\{\beta^{\prime}: \beta^{\prime}=\beta_{i} \text { for some } i \text { s.t. } x \in \alpha_{i} \in \mathscr{A}^{\prime \prime}\right\}
$$

and infer from (1) that

$$
\begin{aligned}
\phi\left(S_{4}\right) & =\int I_{\left(A^{\prime} \cap \sigma \mathscr{A}^{\prime \prime}\right)}(x) \nu\left(x, B-B^{\prime}\right) \mu d x \\
& \leqq \int I_{\sigma \mathscr{A} \prime \prime}(x) \nu\left(x, B-B^{\prime}\right) \mu d x \\
& \leqq \int I_{\sigma \mathscr{A}^{\prime \prime}}(x) \nu(x, B-d(x)) \mu d x \\
& \leqq \int I_{\sigma \mathscr{A}^{\prime \prime}}(x)\left(\frac{r}{3 \mu(A)}\right) \mu d x \\
& \leqq \int I_{A}(x)\left(\frac{r}{3 \mu(A)}\right) \mu d x=r / 3 .
\end{aligned}
$$

Consequently,

$$
\phi(S)<r / 3+0+r / 3+r / 3+\phi(C)<r+\phi(C),
$$

and $\phi(S-C)=\phi(S)-\phi(C)<r$ and since $C$ is clearly a closed subset of $S$ the desired conclusion is at hand.

2.2. LemMA. If $\mathscr{M}$ is a.L. and i.r., $\mathscr{N}$ is a.L. and i.r., $v$ is continuous, each member of $\mathscr{G}$ is an open rectangle in $R, S=\sigma \mathscr{G}$, and $\mu(A)<\infty, \phi(A \times B)<\infty$ for each $A \times B \in \mathscr{G}$, and

$$
\sup _{C \subset S, \phi(C)<\infty ; C \text { closed }} \phi(C)=a<\infty
$$

then

1. $\phi(S)=a$,

2. $S$ is $\phi$ measurable and $\phi(S)<\infty$,

3. there is such a countable subfamily $\mathscr{G}^{\prime}$ of $\mathscr{G}$ that

$$
\phi\left(S-\sigma \mathscr{G}^{\prime}\right)=0 .
$$

Proof. Let

$$
b=\sup _{\mathscr{H} \subset \mathscr{G} ; \mathscr{H} \text { countable }} \phi(\sigma \mathscr{H})
$$

and so choose such a countable subfamily $\mathscr{G}^{\prime}$ of $\mathscr{G}$ that $b=\phi\left(\sigma \mathscr{G}^{\prime}\right)$. We divide the remainder of the proof into 5 steps. The first of these is easily checked with the aid of 2.1 and the second is an immediate consequence of 2.0.

Step 1. $\phi\left(\sigma \mathscr{G}^{\prime}\right)=b \leqq a<\infty, a \leqq \phi(S)$ and $\sigma \mathscr{G}^{\prime}$ is $\phi$ measurable.

Step 2. $\iint I_{S}(x, y) \nu(x, \cdot) d y \mu d x=b$.

Step 3. $\phi\left(S-\sigma \mathscr{G}^{\prime}\right)=0$.

Proof. Let $T=\sigma \mathscr{G}^{\prime}$ and $U=S-T$.

From Step 2 we know

$$
\iint I_{S}(x, y) \nu(x, \cdot) d y \mu d x=b
$$


On the other hand from Step 1 and 1.6 we learn .5

$$
\iint I_{T}(x, y) v(x, \cdot) d y \mu d x=b .
$$

Now since, for each $z$

$$
I_{U}(z)=I_{S}(z)-I_{T}(z)
$$

we infer from $.4, .5$ and Step 1 that

$$
\iint I_{U}(x, y) \nu(x, \cdot) d y \mu d x=0 .
$$

Thus $U$ is a nilset, and because of 1.0.4 and 1.0.3, $\phi(U)=0$.

Step 4. $S$ is $\phi$ measurable and $\phi(S)=b$.

Proof. Use Steps 1 and 3.

Step 5. $\phi(S)=a$.

Proof. With the help of Steps 1 and 4 conclude $\phi(S)=b \leqq a \leqq \phi(S)$.

The fundamental result of this section, namely the preservation of the topological properties of a.L. and i.r. under the condition that $\nu$ is continuous and $M$ and $R$ have finite measure is given in our last

2.3. Theorem. If $\mathscr{M}$ is a.L. and i.r., $\mathscr{N}$ is a.L. and i.r., $\nu$ is continuous, and $\mu(M)<\infty$ and $\phi(R)<\infty$ then $\mathscr{T}$ is a.L. and i.r.

Proof. From 2.2.2 we learn that the members of $\mathscr{T}$ are $\phi$ measurable. Taking $S=R$ in 2.2 shows (in .3) that if $\mathscr{G}$ is any open covering of $R$ then there is such a countable subfamily $\mathscr{G}^{\prime}$ of $\mathscr{G}$ that $\phi\left(R-\sigma^{\prime} \mathscr{G}^{\prime}\right)=0$. Hence $\mathscr{T}$ is a.L. From 2.2 .1 we conclude $\mathscr{T}$ is i.r. to complete the proof.

The condition above concerning finite measure can be relaxed in at least two ways. One obvious way attempts to replace finiteness with some kind of $\sigma$-finiteness requirement. An alternate way, like that taken in PM, removes the restriction entirely. This method requires modifications of our definitions of the topological properties and also involves a certain artificiality to achieve an approximation that was natural in the case of product measures. For these reasons it is not developed here.

3. Acknowledgement. We should point out that the material in $\$ 1$, although organized differently than $\S 5$ of PM, is essentially taken therefrom by replacing the occurrences of " $\nu$ " with " $\nu(x, \cdot)$." This is also true of Lemma 2.0 which comes from PM 7.4 and 2.2 which comes from PM 7.5. It would be desirable to use the results of PM (with some sort of transformation) to obtain the results given here rather than to reformulate the proofs of PM as we have done. But we were unable to find a way to accomplish it.

\section{REFERENCE}

1. W. W. Bledsoe and A. P. Morse, Product measures, Trans. Amer. Math. Soc. 79 (1955), 173-215.

Bell Telephone Laboratories, Inc., HOLMDEL, NEW JERSEY 